\title{
Several reactions of tantalum-imine complexes with isocyanates, a hydrazone, and imides
}

\author{
Haruka Shimizu, Miho Ohsumi, and Shu Kobayashi*
}

Graduate School of Pharmaceutical Sciences, The University of Tokyo, The HFRE Division, ERATO, Japan Science and Technology Agency (JST), Hongo, Bunkyo-ku, Tokyo 113-0033,

\author{
Japan \\ E-mail: skobayas@mol.f.u-tokyo.ac.jp
}

This paper is dedicated to Professor Eusebio Juaristi on the occasion of his $55^{\text {th }}$ birthday

(received 24 Aug 05; accepted 26 Oct 05; published on the web 02 Dec 05)

\begin{abstract}
We have revealed that tantalum-imine complexes reacted regioselectively with phenyl or benzyl isocyanate to afford $\alpha$-amino amides in high yields. The reaction provides a general method of $\alpha$ amino amides from various imines and isocyanates using a low-valent tantalum prepared from $\mathrm{TaCl}_{5}$ and $\mathrm{Zn}$ in situ. In addition, we have found a novel reaction of a tantalum-imine complex with a hydrazone and imides to afford heterocyclic compounds in good yields.
\end{abstract}

Keywords: Tantalum-imine complex, isocyanate, $\alpha$-amino amides, $N$-methyl imides, hydrazone

\section{Introduction}

Metal-imine complexes have attracted much attention in the area of organometallic chemistry, and their formation and chemical properties have been well investigated. ${ }^{1-4}$ In many cases, they were formed in situ via ligand-metal exchange followed by a loss of $\beta$-hydrogen of an amide moiety, ${ }^{5}$ migration of an alkyl group to an (iminoacyl)metal, ${ }^{6}$ and reduction of a low-valent complex with phosphaazaallene. ${ }^{7}$ In the last decade, several examples of convenient syntheses of metal-imine complexes, which were formed by direct reactions of imines with metals or metal salts, have been reported. ${ }^{8}$ As for group 5 metals, many niobium-imine and tantalum-imine complexes have been postulated as intermediates in several organometallic transformations, ${ }^{3}$ whereas only a few have been isolated and crystallographically characterized. ${ }^{9}$ In 1987 , Pedersen et al. reported that treatment of aldimines with $\mathrm{NbCl}_{3}(\mathrm{dme})$ followed by carbonyl compounds gave $\beta$-amino alcohols in good yields. ${ }^{3 e}$ They discussed in the paper that these reactions 
proceeded via niobium-imine complexes although their characteristic structures were not confirmed. In 1998, Takai et al. reported the first example of direct synthesis of tantalum-imine complex (2) from a low-valent tantalum prepared from $\mathrm{TaCl}_{5}$ and $\mathrm{Zn}$ and an imine. ${ }^{8 \mathrm{a}}$ They also disclosed its characteristic structure and reactivity toward carbonyl compounds and an isocyanate. We are interested in the synthetic utilities of $\mathbf{2}$ and its analogues because their use in organic syntheses has been rather limited.

Whereas a wide variety of methods are available for the syntheses of $\alpha$-amino acids and their analogues, ${ }^{10}$ development of new and more general approaches is still an important task. The reactions of tantalum-imine complexes with isocyanates might provide a useful synthetic method for $\alpha$-amino amides if isocyanates inserted into tantalum-imine complexes regioselectively. Moreover, there is a possibility that tantalum-imine complexes react with various electrophiles other than aldehydes, ketones, and isocyanates. Therefore, we started to investigate further possibility of tantalum-imine complexes as important synthetic intermediates.

\section{Results and Discussion}

\section{Reactions with isocyanates}

We thought that a tantalum-imine complex could be a general precursor for $\alpha$-amino amides. It was reported that 2 reacted with cyclohexyl isocyanate to afford a mixture of $\alpha$-amino amide and a urea derivative in almost $2: 1$ ratio (Scheme 1). ${ }^{8 a}$
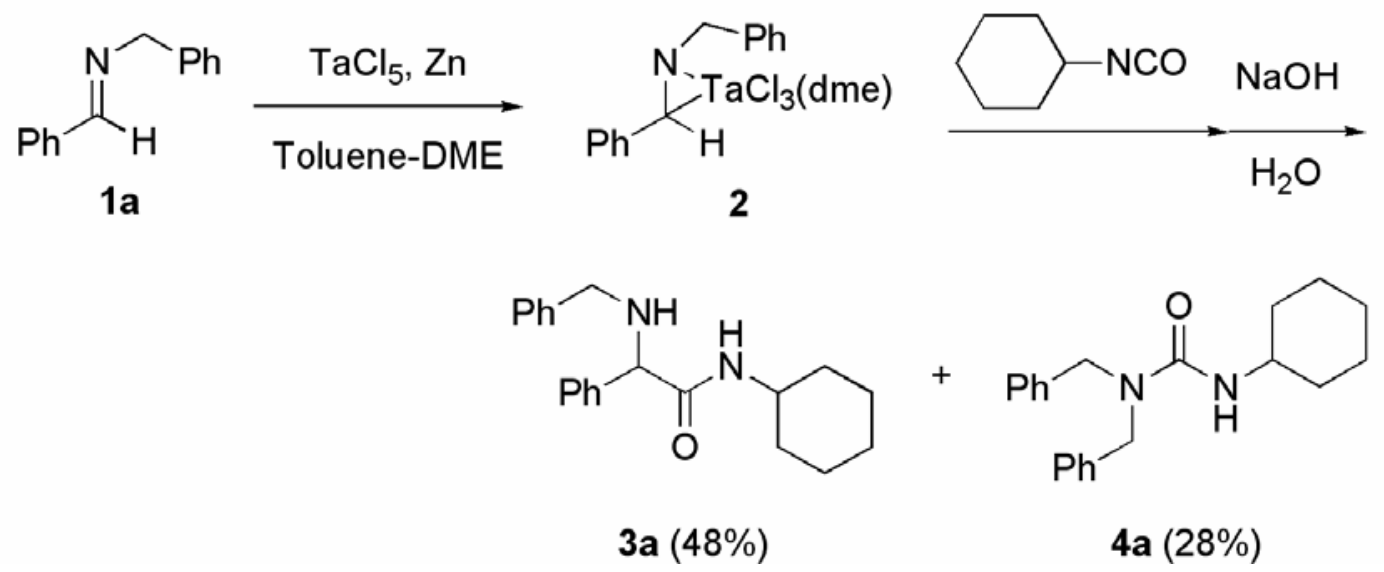

Scheme 1. The reaction of a Ta-imine complex with cyclohexyl isocyanate.

On the other hand, it was known in the reaction of a zirconium-imine complex with isocyanate that steric hindrance on the imine nitrogen or on the isocyanate discouraged insertion into the $\mathrm{Zr}$ $\mathrm{N}$ bond and gave clean $\mathrm{Zr}-\mathrm{C}$ insertion. ${ }^{5 \mathrm{c}}$ Since it was expected that the same phenomena could be observed in the case of a tantalum-imine complex, we used phenyl isocyanate instead of 
cyclohexyl isocyanate. The reaction proceeded to give the desired $\alpha$-amino amide although the yield was low (Table 1, entry 1). The reaction system was rather messy, and it was found that the use of an excess amount of the isocyanate decreased the yield of the desired product and led to an increase of unknown insoluble by-products (Table 1, entry 2). We then decided to change the work up procedure to more harsh conditions, which meant using aqueous $10 \% \mathrm{KOH}$ and stirring for $1 \mathrm{~h}$ at room temperature, in order to deactivate the unreacted isocyanate. As expected, the yield was improved (Table 1, entry 3 ) although a by-product, which was thought to be a urea derivative of the product, was still obtained. Further optimization of the reaction conditions revealed that the use of a slight excess of the imine led to an increased yield, up to $90 \%$ in $24 \mathrm{~h}$ (entry 5). Benzyl isocyanate also gave a promising result (entry 7). In this case, phenyl and benzyl isocyanates seemed more suitable than cyclohexyl isocyanate.

Table 1. Optimization of the reaction conditions

\begin{tabular}{|c|c|c|c|c|c|c|}
\hline & $\mathrm{Ph}$ & $\mathrm{R}-\mathrm{NCO}$ & $\begin{array}{l}\mathrm{Cl}_{5}(1 \mathrm{equ} \\
\text { (1.2 equiv } \\
\text { zzene-DM }\end{array}$ & quench & $\mathrm{P}$ & $\prod_{5}^{\mathrm{N}}$ \\
\hline entry & 1a (equiv) & $\mathrm{R}-\mathrm{NCO}$ (equiv) & time (h) & quench & product & yield (\%) \\
\hline 1 & 1.0 & PhNCO (1.2) & 18 & sat. $\mathrm{NaHCO}_{3}$ aq. & $5 a$ & 37 \\
\hline 2 & 1.0 & PhNCO (2.4) & 18 & sat. $\mathrm{NaHCO}_{3}$ aq. & $5 a$ & complex mixture \\
\hline 3 & 1.0 & $\mathrm{PhNCO}(1.2)$ & 18 & $10 \% \mathrm{KOH}$ aq. & $5 a$ & 78 \\
\hline 4 & 1.2 & PhNCO (1.0) & 18 & $10 \% \mathrm{KOH}$ aq. & $5 a$ & 82 \\
\hline 5 & 1.2 & PhNCO (1.0) & 24 & $10 \% \mathrm{KOH}$ aq. & $5 a$ & 90 \\
\hline 6 & 1.2 & $\mathrm{PhNCO}(1.0)$ & 48 & $10 \% \mathrm{KOH}$ aq. & $5 a$ & 82 \\
\hline 7 & 1.2 & $\mathrm{BnNCO}(1.0)$ & 24 & $10 \% \mathrm{KOH}$ aq. & $5 b$ & 90 \\
\hline
\end{tabular}

Encouraged by these results, we next investigated the substrate scope of this reaction (Table 2). In the cases of $N$-(4-substituted-benzylidene)benzylamine derivatives, the desired products were obtained in high yields (entries 1-3, 9-11), although the formation of Ta-imine complexes from benzylamine derivatives with electron-withdrawing substituents proceeded slowly. $\mathrm{N}$ Benzylidenebenzylaniline and aldimines prepared from heteroaromatic aldehydes and benzylamine also required longer reaction time to form the corresponding Ta-imine complexes in good yields. The aliphatic imine derived from cyclohexanecarboxyaldehyde, and the ketimine derived from cyclohexanone also worked well to afford the corresponding products in good or moderate yields, respectively (Table 2, entry 14 , Scheme 2). 

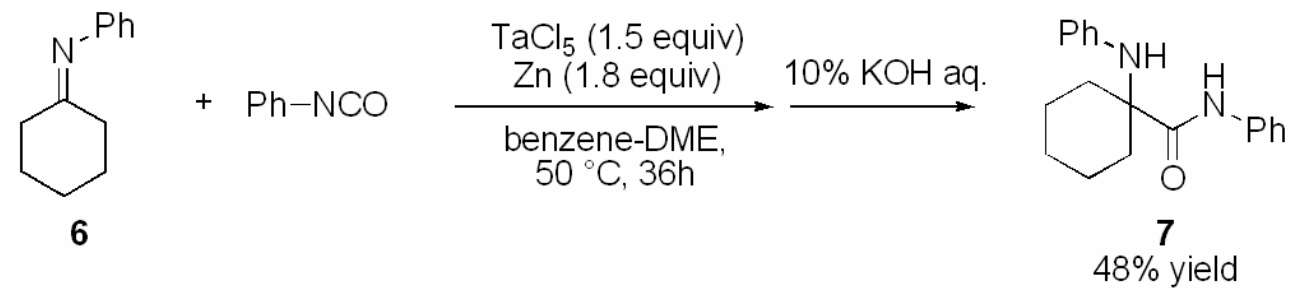

Scheme 2. The reaction of the ketimine with isocyanate.

In addition, it was found that aldimines derived from chiral amines moderately influenced the stereochemical outcome of subsequent additions of Ta-imine complexes to isocyanates (Table 2, entries 6-8, 15-17).

Table 2. Substrate scope

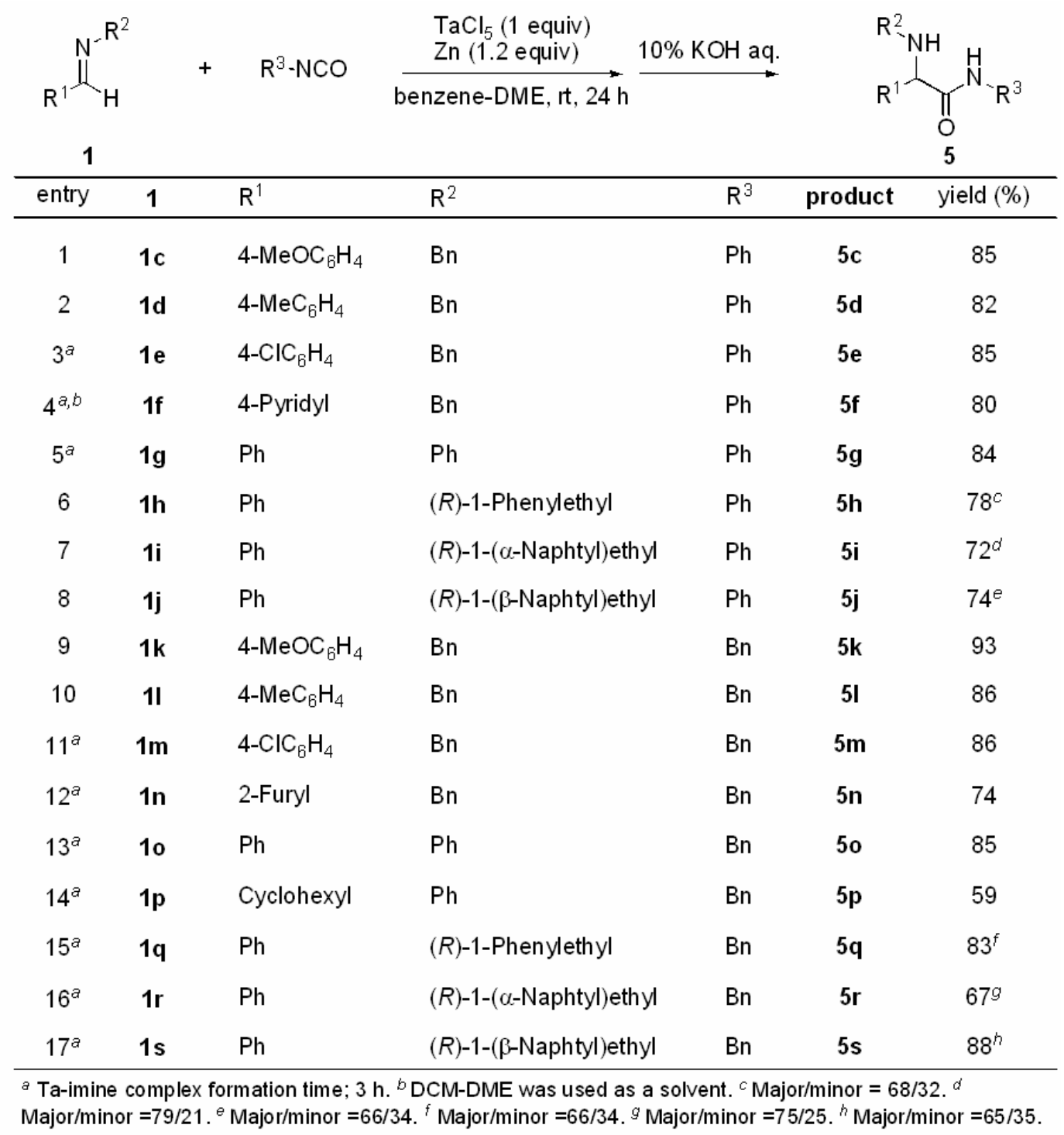


These results suggested that this strategy might provide a general preparation method for natural and unnatural $\alpha$-amino amides including $\alpha, \alpha$-disubstituted $\alpha$-amino amides. ${ }^{11,12}$ Further investigation into asymmetric version of this reaction is in progress.

\section{Reactions with a hydrazone and imides}

Next we turned our attention to reactions of $\mathbf{2}$ with carbonyl compounds. It was also reported that 2 reacted with carbonyl compounds to afford $\beta$-amino alcohols in good yields. On the other hand, it was revealed by Arai et al. that homo-pinacol coupling of an imine proceeded to give diamine derivalites in good yield using a low-valent niobium prepared from $\mathrm{NbCl}_{5}$ and $\mathrm{Zn}^{13}$ Although it was known that 2 did not react with imines, it was thought that it might react with imine equivalents such as hydrazones to give diamine equivalents. Acylhydrazones ${ }^{14}$ are known to be useful imine equivalents because they are solids in many cases and very stable even in the cases of acylhydrazones derived from aliphatic imines. Therefore, we decided to conduct a cross coupling reaction of 2 that was prepared in situ with the benzoylhydrazone derived from hydrocinnamaldehyde. It turned out that the reaction proceeded to give a crystalline compound, whose structure was determined by X-ray crystal structural analysis (Scheme 3 ). ${ }^{15}$
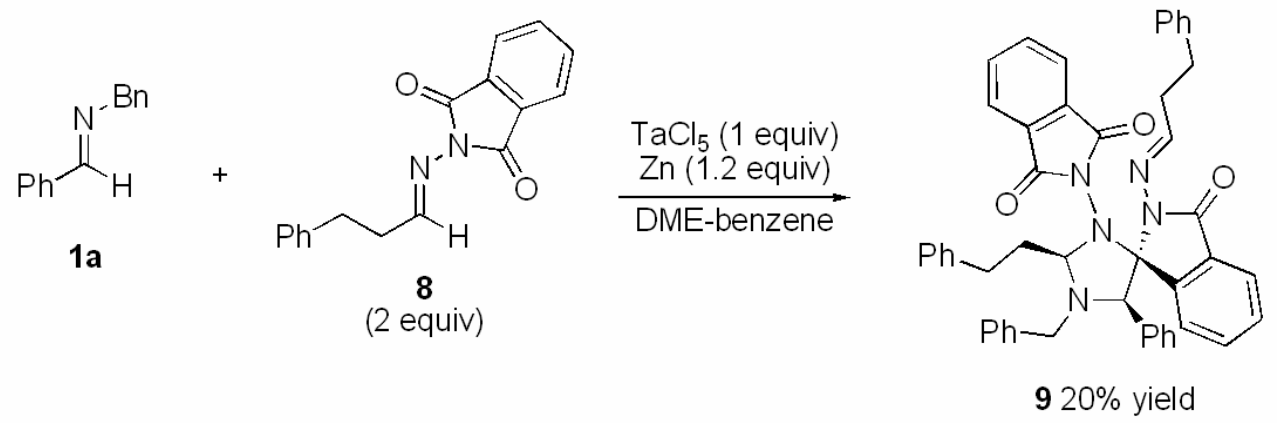

Scheme 3. The reaction of Ta-imine complex with benzoylhydrazone. 


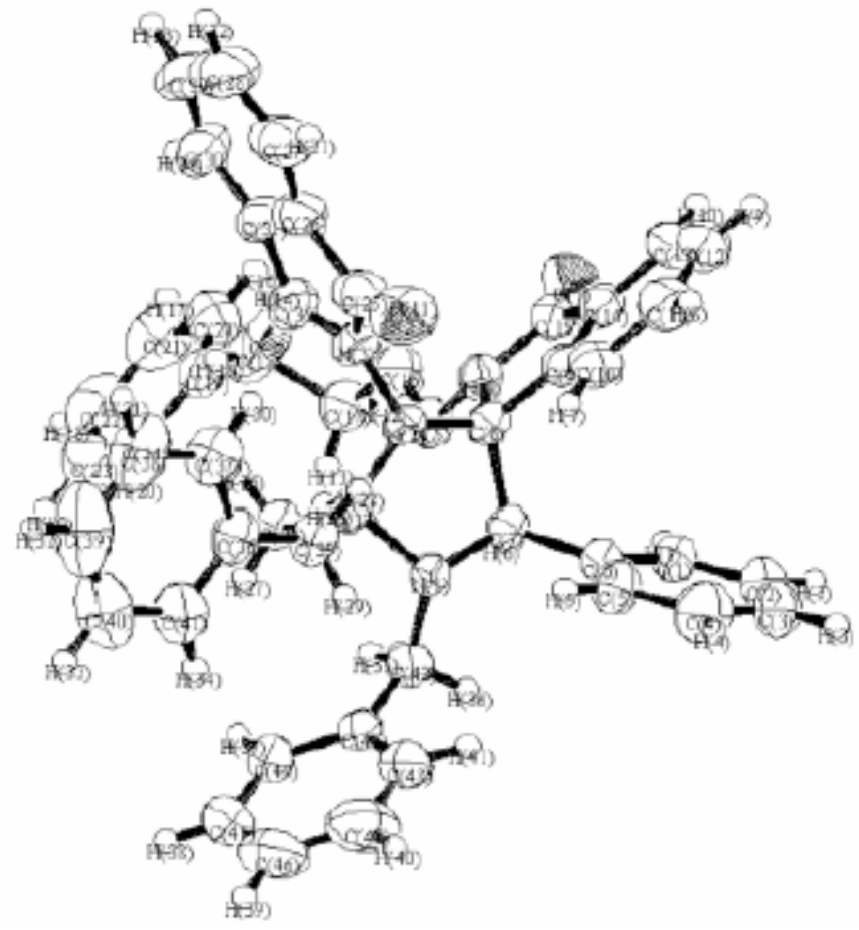

Figure 1. Ortep drawing of 9.

The compound has a highly complicated structure, which is composed of one imine and two benzoylhydrazones (Figure 1). This odd molecule inspired us to try reactions of tantalum-imine complexes with imides to afford interesting heterocyclic compounds. Indeed, the reactions of a tantalum-imine complex with $N$-methylphthalimide (10a) and $N$-methylsucciimide (10b) gave highly functionalized heterocyclic compounds in moderate yields with moderate to good diastereoselectivities, although $N$-benzylmaleimide (10c) resulted in the formation of a complex mixture (Table 3). 
Table 3. Reactions of Ta-imine complex with imides

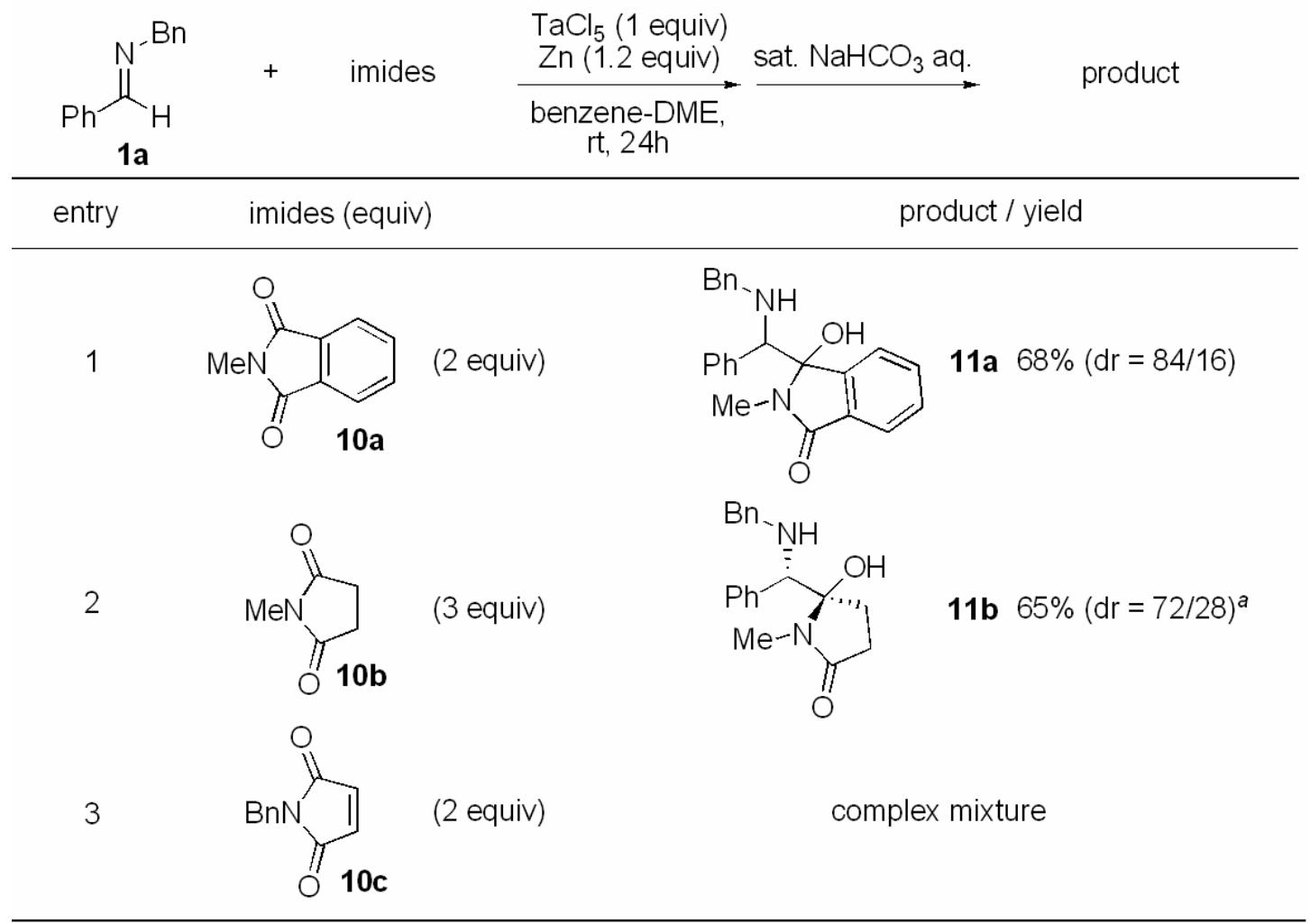

${ }^{a}$ Relative configuration of the major diastereoisomer was determined by X-ray structural analysis.

\section{Conclusions}

In summary, we have demonstrated the synthetic utilities of tantalum-imine complexes for the syntheses of $\alpha$-amino amides and heterocyclic compounds. It was found that $\alpha$-amino amides were readily prepared from imines and isocyanates using a low-valent tantalum generated from $\mathrm{TaCl}_{5}$ and $\mathrm{Zn}$. A wide variety of imines and isocyanates were applicable, and various $\alpha$-amino amides were synthesized in high yields. In addition, the first example of the reactions of tantalum-imine complexes with imides was reported, which gave heterocyclic derivatives. These results suggested further applicability of tantalum-imine complexes as useful intermediates in organic synthesis. Further investigations along this line are now in progress.

\section{Experimental Section}

General Procedures. Melting points were uncorrected. IR spectra were measured with JASCO FT/IR-610 spectrometers. ${ }^{1} \mathrm{H}$ and ${ }^{13} \mathrm{C}$ NMR spectra were recorded on a JEOL JNM-LA300, JNM-LA400, ECX-400 or ECX-600 spectrometer in $\mathrm{CDCl}_{3}$ unless otherwise noted. 
Tetramethylsilane (TMS) served as an internal standard $(\delta=0 \mathrm{ppm})$ for ${ }^{1} \mathrm{H} \mathrm{NMR}$ and $\mathrm{CDCl}_{3}$ as an internal standard $(\delta=77.0 \mathrm{ppm})$ for ${ }^{13} \mathrm{C}$ NMR. FAB-MS and ESI-MS spectra were measured with JEOL JMS-MS700V and Bruker Daltonics BioTOF II, respectively. X-ray crystal structure analysis was performed on a Rigaku R-AXIS RAPID AUTO. Preparative thin-layer chromatography was carried out using Wakogel B-5F. 1,2-Dimethoxyethane (DME) was distilled from ketyl and stored with $\mathrm{Na}$ under argon. Dehydrated benzene was purchased from Wako Pure Chemical Industries Ltd. Zinc powder was purchased from Nacalai Tesque and used after washing with $1 \mathrm{~N} \mathrm{HCl}$, water, and ethanol successively and dried at $200{ }^{\circ} \mathrm{C}$ for $12 \mathrm{~h}$.

\section{Typical experimental procedure}

(Table 1, entry 5). Under argon atmosphere, DME $(0.25 \mathrm{ml})$ was added to a suspension of $\mathrm{TaCl}_{5}$ $(144 \mathrm{mg}, 0.40 \mathrm{mmol})$ and $\mathrm{Zn}(32 \mathrm{mg}, 0.49 \mathrm{mmol})$ in benzene $(0.25 \mathrm{ml})$, and the mixture was stirred at room temperature for $1 \mathrm{~h}$. $N$-Benzylidenebenzylamine (1a) (93 $\mathrm{mg}, 0.48 \mathrm{mmol}$ ) in benzene $(0.50 \mathrm{ml})$ was added to the resulting green-brown suspension at the same temperature, and the mixture was stirred for $1 \mathrm{~h}$. The color of the suspension changed to dark red. Phenyl isocyanate $(48 \mathrm{mg}, 0.40 \mathrm{mmol})$ in benzene $(0.50 \mathrm{ml})$ was then added, and the mixture was stirred at room temperature for $24 \mathrm{~h}$. The reaction was stopped by addition of aqueous $10 \% \mathrm{KOH}(5 \mathrm{ml})$ and ether $(5 \mathrm{ml})$, and the mixture was stirred vigorously for $30 \mathrm{~min}$ until the brown mixture became white suspension. The white solid was filtered off through celite ${ }^{\circledR}$ and the residue was washed with ether $(10 \mathrm{ml}, 3$ times). The filtrate and the washes were combined and extracted with ether (10 ml, 2 times). The organic layer was combined and washed with brine and dried over $\mathrm{Na}_{2} \mathrm{SO}_{4}$. The crude product was purified by P-TLC $\left(\mathrm{CH}_{2} \mathrm{Cl}_{2}-\mathrm{EtOH}, 30: 1\right)$ to afford 2(benzylamino)- $N$,2-diphenylacetamide (5a) (115 mg, 90\%).

\section{Compound characterization}

2-(Benzylamino)- $N$,2-diphenylacetamide (5a). ${ }^{1} \mathrm{H} \mathrm{NMR}\left(400 \mathrm{MHz}, \mathrm{CDCl}_{3}\right) \delta 3.85$ (s, 2H), $4.33(\mathrm{~s}, 1 \mathrm{H}), 7.01-7.60(\mathrm{~m}, 15 \mathrm{H}), 9.47$ (brs, $1 \mathrm{H}) ;{ }^{13} \mathrm{C} \mathrm{NMR}\left(400 \mathrm{MHz}, \mathrm{CDCl}_{3}\right) \delta 52.7,67.5$, 119.4, 124.1, 127.2, 127.5, 128.1, 128.7, 128.8, 128.9, 137.6, 138.8, 170.0; IR (KBr) 3303, 3261, 1672, 1603, 1533, 1495, 1443, 1119, 924, 739, $694 \mathrm{~cm}^{-1}$; ESIMS m/z $317([\mathrm{M}+\mathrm{H}]+)$; Anl. Calcd for $\mathrm{C}_{21} \mathrm{H}_{20} \mathrm{~N}_{2} \mathrm{O}$ : C, 79.44; H, 6.00; N, 9.26. Found: C, 79.50; H, 6.20; N, 9.22; Mp: 80-82 ${ }^{\circ} \mathrm{C}$.

$\boldsymbol{N}$-Benzyl-2-(benzylamino)-2-phenylacetamide (5b). ${ }^{16}{ }^{1} \mathrm{H} \mathrm{NMR}\left(\mathrm{CDCl}_{3}\right) \delta 2.01$ (brs, $\left.1 \mathrm{H}\right), 3.76$ $(\mathrm{dd}, 2 \mathrm{H}, J=13,16 \mathrm{~Hz}), 4.29(\mathrm{~s}, 1 \mathrm{H}), 4.45(\mathrm{ddd}, 2 \mathrm{H}, J=6.2,15,21 \mathrm{~Hz}), 7.20-7.48(\mathrm{~m}, 14 \mathrm{H})$, $7.49(\mathrm{~m}, 1 \mathrm{H}) ;{ }^{13} \mathrm{C} \mathrm{NMR}\left(\mathrm{CDCl}_{3}\right) \delta 43.1,52.5,66.9,127.3,127.4,127.6,128.1,128.5,128.6$, $128.8,138.3,139.1,139.1,171.9$.

2-(Benzylamino)-2-(4-methoxyphenyl)- $\boldsymbol{N}$-phenylacetamide (5c). ${ }^{1} \mathrm{H} \mathrm{NMR}\left(\mathrm{CDCl}_{3}\right) \delta 2.03$ (brs, 1H), $3.75(\mathrm{~s}, 3 \mathrm{H}), 3.84(\mathrm{~s}, 2 \mathrm{H}), 4.28(\mathrm{~s}, 1 \mathrm{H}), 6.85(\mathrm{~m}, 2 \mathrm{H}), 7.08(\mathrm{~m}, 1 \mathrm{H}), 7.25-7.37(\mathrm{~m}, 9 \mathrm{H})$, $7.56(\mathrm{~m}, 2 \mathrm{H}), 9.35(\mathrm{~s}, 1 \mathrm{H}) ;{ }^{13} \mathrm{C} \mathrm{NMR}\left(\mathrm{CDCl}_{3}\right) \delta 52.6,55.2,66.9,114.3,119.4,124.1,127.4$, $128.1,128.3,128.6,128.9,131.0,137.6,138.9,159.5,170.4$; IR (neat) 3293, 3032, 1679, 1601, 
1512, 1440, 1249, 1176, 755, 696; HRMS (ESI-TOF) calcd for $\mathrm{C}_{22} \mathrm{H}_{23} \mathrm{~N}_{2} \mathrm{O}_{2}([\mathrm{M}+\mathrm{H}]+)$ : 347.1754, found: 347.1753 .

2-(Benzylamino)- $\boldsymbol{N}$-phenyl-2-p-tolylacetamide (5d). ${ }^{1} \mathrm{H}$ NMR $\left(\mathrm{CDCl}_{3}\right) \delta 2.21$ (s, 3H), 3.74 (s, 2H), 4.20 (s, 1H), 6.96-7.00 (m, 3H), 7.00-7.27 (m, 9H), 7.47 (d, 2H, J=8.2 Hz), $9.27(\mathrm{~s}, 1 \mathrm{H})$; ${ }^{13} \mathrm{C} \mathrm{NMR}\left(\mathrm{CDCl}_{3}\right) \delta 21.0,52.6,67.2,119.4,124.1,127.0,127.4,128.1,128.6,128.9,129.5$, 135.9, 137.6, 138.0, 138.9, 170.2; IR (neat) 3234, 1677, 1600, 1519, 1440, 755, 695; HRMS (ESI-TOF) calcd for $\mathrm{C}_{22} \mathrm{H}_{23} \mathrm{~N}_{2} \mathrm{O}([\mathrm{M}+\mathrm{H}]+)$ : 331.1805 , found: 331.1800 .

2-(Benzylamino)-2-(4-chlorophenyl)- $\mathrm{N}$-phenylacetamide (5e). ${ }^{1} \mathrm{H}$ NMR $\left(\mathrm{CDCl}_{3}\right) \delta 2.26$ (brs, $1 \mathrm{H}), 3.83$ (s, 2H), 4.29 (s, 1H), 7.09 (t, 1H, J=7.1 Hz), 7.11-7.37 (m, 11H), 7.55 (m, 2H), 9.35 $(\mathrm{s}, 1 \mathrm{H}) ;{ }^{13} \mathrm{C} \mathrm{NMR}\left(\mathrm{CDCl}_{3}\right) \delta 52.5,66.7,119.4,124.3,127.6,128.1,128.5,128.7,129.0,129.0$, 134.1, 137.2, 137.4, 138.6, 169.6; IR (KBr) 3298, 1658, 1599, 1521, 1443, 1090, 829, 750; HRMS (ESI-TOF) calcd for $\mathrm{C}_{21} \mathrm{H}_{20} \mathrm{ClN}_{2} \mathrm{O}([\mathrm{M}+\mathrm{H}]+)$ : 351.1259 , found: 351.1263 ; Mp: $97-99{ }^{\circ} \mathrm{C}$. 2-(Benzylamino)- $\boldsymbol{N}$-phenyl-2-(pyridin-4-yl)acetamide (5f). ${ }^{1} \mathrm{H} \mathrm{NMR}\left(\mathrm{CDCl}_{3}\right) \delta 3.68$ (dd, $2 \mathrm{H}, J$ $=13,16 \mathrm{~Hz}), 4.32(\mathrm{~s}, 1 \mathrm{H}), 7.11(\mathrm{~m}, 1 \mathrm{H}), 7.25-7.38(\mathrm{~m}, 9 \mathrm{H}), 7.54(\mathrm{~m}, 2 \mathrm{H}), 8.54(\mathrm{~m}, 2 \mathrm{H}), 9.34(\mathrm{~s}$, $1 \mathrm{H}) ;{ }^{13} \mathrm{C} \mathrm{NMR}\left(\mathrm{CDCl}_{3}\right) \delta 52.5,66.3,119.5,122.2,124.6,127.8,128.2,128.8,129.0,137.2$, 138.3, 147.3, 150.2, 168.6; IR (neat) 3200, 3038, 1686, 1600, 1521, 1442, 754, 735, 696; HRMS (FABMS) calcd for $\mathrm{C}_{20} \mathrm{H}_{20} \mathrm{~N}_{3} \mathrm{O}([\mathrm{M}+\mathrm{H}]+)$ : 318.1606 , found: 318.1601 .

$\mathbf{N}$,2-Diphenyl-2-(phenylamino)acetamide (5g). ${ }^{5 \mathrm{c}}{ }^{1} \mathrm{H}$ NMR $\left(\mathrm{CDCl}_{3}\right) \delta 4.51$ (brs, $\left.1 \mathrm{H}\right), 4.81$ (s, $1 \mathrm{H}), 6.66(\mathrm{~m}, 2 \mathrm{H}), 6.82(\mathrm{~m}, 1 \mathrm{H}), 7.04-7.49(\mathrm{~m}, 12 \mathrm{H}), 8.82(\mathrm{~s}, 1 \mathrm{H}) ;{ }^{13} \mathrm{C} \mathrm{NMR}\left(\mathrm{CDCl}_{3}\right) \delta 65.1$, $114.0,119.6,119.9,124.5,127.3,128.7,128.9,129.2,129.4,137.2,138.3,146.4,169.6$.

2-((R)-1-Phenylethylamino)- $N$,2-diphenylacetamide (5h). ${ }^{1} \mathrm{H}$ NMR $\left(\mathrm{CDCl}_{3}\right)$ [minor isomer] $\delta$ $1.47(\mathrm{~d}, 3 \mathrm{H}, J=6.8 \mathrm{~Hz}), 3.86(\mathrm{dd}, 1 \mathrm{H}, J=6.8,13 \mathrm{~Hz}), 4.16(\mathrm{~s}, 1 \mathrm{H}), 7.11(\mathrm{~m}, 1 \mathrm{H}), 7.23-7.37(\mathrm{~m}$, $12 \mathrm{H}), 7.64(\mathrm{~m}, 2 \mathrm{H}), 9.58(\mathrm{~s}, 1 \mathrm{H})$; [major isomer] $\delta 1.43(\mathrm{~d}, 3 \mathrm{H}, J=6.8 \mathrm{~Hz}), 3.77(\mathrm{dd}, 1 \mathrm{H}, J=$ 6.8, $13 \mathrm{~Hz}), 4.22(\mathrm{~s}, 1 \mathrm{H}), 7.03-7.45(\mathrm{~m}, 15 \mathrm{H}), 9.11(\mathrm{~s}, 1 \mathrm{H}) ;{ }^{13} \mathrm{C} \mathrm{NMR}\left(\mathrm{CDCl}_{3}\right)$ [minor isomer] $\delta$ 24.0, 57.6, 65.7, 119.4, 124.2, 126.5, 127.2, 127.6, 128.2, 128.8, 129.0, 129.1, 137.7, 170.4; [major isomer] $\delta 23.7,56.5,65.8,119.3,124.0,126.6,127.4,127.5,128.1,128.7,128.8,128.9$, 137.5, 139.3, 144.0, 170.2; IR (neat) [major isomer] 3234, 3059, 1686, 1600, 1523, 1435, 1265 , 744, 704; HRMS (ESI-TOF) calcd for $\mathrm{C}_{22} \mathrm{H}_{23} \mathrm{~N}_{2} \mathrm{O}([\mathrm{M}+\mathrm{H}]+)$ : 331.1805 , found: 331.1801 .

2-((R)-1-(Naphthalen-1-yl)ethylamino)- $N$,2-diphenylacetamide (5i). ${ }^{1} \mathrm{H} \quad \mathrm{NMR} \quad\left(\mathrm{CDCl}_{3}\right)$ [mixture] $\delta 1.56(\mathrm{~m}, 3 \mathrm{H}), 2.36(\mathrm{brs}, 1 \mathrm{H}), 4.18(\mathrm{~s}, 0.22 \mathrm{H}), 4.32(\mathrm{~s}, 0.78 \mathrm{H}), 4.58(\mathrm{dd}, 0.78 \mathrm{H}, \mathrm{J}=$ 6.9, $13 \mathrm{~Hz}), 4.70(\mathrm{dd}, 0.22 \mathrm{H}, \mathrm{J}=6.4,13 \mathrm{~Hz}), 6.99-8.07(\mathrm{~m}, 17 \mathrm{H}), 9.09(\mathrm{~s}, 0.78 \mathrm{H}), 9.48$ (s, $0.22 \mathrm{H}) ;{ }^{13} \mathrm{C} \mathrm{NMR}\left(\mathrm{CDCl}_{3}\right)$ [mixture] $\delta 23.3,23.4,52.4,52.5,65.8,65.9,119.3,119.4,122.5$, $122.8,123.3,124.0,124.1,125.4,125.5,125.6,125.6,126.0,126.2,127.1,127.6,127.8,128.1$, $128.1,128.7,128.9,128.9,129.0,129.1,130.1,133.9,134.1,137.4,137.6,139.4,139.7,139.8$, 170.3, 170.7; IR (KBr) 3327, 1678, 1600, 1523, 1442, 752, 697; HRMS (FABMS) calcd for C26H25N2O ([M+H]+): 381.1967, found: 381.1974 .

2-((R)-1-(Naphthalen-3-yl)ethylamino)- $\boldsymbol{N}$,2-diphenylacetamide $\quad \mathbf{( 5 j}) .{ }^{1} \mathrm{H} \quad \mathrm{NMR} \quad\left(\mathrm{CDCl}_{3}\right)$ [mixture] $\delta 1.50(\mathrm{~m}, 3 \mathrm{H}), 2.26(\mathrm{brs}, 1 \mathrm{H}), 3.90(\mathrm{dd}, 0.7 \mathrm{H}, J=6.9,13 \mathrm{~Hz}), 3.98(\mathrm{dd}, 0.3 \mathrm{H}, J=6.9$, 
$13 \mathrm{~Hz}), 4.15(\mathrm{~s}, 0.3 \mathrm{H}), 4.25(\mathrm{~s}, 0.7 \mathrm{H}), 7.00-7.82(\mathrm{~m}, 16 \mathrm{H}), 9.04(\mathrm{~s}, 0.7 \mathrm{H}), 9.52(\mathrm{~s}, 0.3 \mathrm{H}) ;{ }^{13} \mathrm{C}$ NMR $\left(\mathrm{CDCl}_{3}\right)$ [mixture] $\delta 23.6,24.0,56.5,57.6,65.6,65.7,119.3 .119 .4,123.9,124.0,124.2$, $124.5,125.5,125.6,125.7,125.8,126.1,126.2$, 127.1, 127.6, 127.6, 127.7, 127.7, 128.1, 128.6, $128.7,128.8,128.8,128.9,132.9,133.3,133.3,137.5,137.7,139.2,139.3,141.1,141.3,170.2$, 170.5; IR (neat) 3314, 1678, 1600, 1523, 1441, 735; HRMS (ESI-TOF) calcd for $\mathrm{C}_{26} \mathrm{H}_{25} \mathrm{~N}_{2} \mathrm{O}$ $([\mathrm{M}+\mathrm{H}]+): 381.1967$, found: 381.1965 .

$\boldsymbol{N}$-Benzyl-2-(benzylamino)-2-(4-methoxyphenyl)acetamide (5k). ${ }^{1} \mathrm{H}$ NMR $\left(\mathrm{CDCl}_{3}\right) \delta 1.93$ (brs, 1H), 3.75 (m, 2H), 3.79 (s, 3H), $4.24(\mathrm{~s}, 1 \mathrm{H}), 4.45$ (d, 2H, J=6.2 Hz), 6.81-6.84 (m, 2H), 7.21-7.32 (m, 12H), 7.43 (brs, $1 \mathrm{H}) ;{ }^{13} \mathrm{C} \mathrm{NMR}\left(\mathrm{CDCl}_{3}\right) \delta 43.2,52.4,55.3,66.3,114.2,127.3$, $127.4,127.6,128.1,128.5,128.5,128.7,131.1,138.3,138.4,153.2,172.2$; IR (neat) 3309, 1659, 1509, 1455, 698; HRMS (FAB) calcd for $\mathrm{C}_{23} \mathrm{H}_{25} \mathrm{~N}_{2} \mathrm{O}_{2}([\mathrm{M}+\mathrm{H}]+)$ ): 361.1911, found: 361.1903 .

$\boldsymbol{N}$-Benzyl-2-(benzylamino)-2-p-tolylacetamide (5l). ${ }^{1} \mathrm{H} \mathrm{NMR}\left(\mathrm{CDCl}_{3}\right) \delta 1.95$ (brs, $\left.1 \mathrm{H}\right), 2.33$ (s, $3 \mathrm{H}), 3.74$ (dd, 2H, $J=13,19 \mathrm{~Hz}), 4.25(\mathrm{~s}, 1 \mathrm{H}), 4.44(\mathrm{~m}, 2 \mathrm{H}), 7.05-7.30$ (m, 14H), 7.45 (brs, 1H); ${ }^{13} \mathrm{C} \mathrm{NMR}\left(\mathrm{CDCl}_{3}\right) \delta 21.1,43.2,52.5,66.7,127.2,127.3,127.4,127.7,128.1,128.6,128.7$, 129.5, 136.2, 138.0, 138.4, 139.2, 172.1; IR (neat) 3309, 1659, 1509, 1455, 698; HRMS (FAB) calcd for $\mathrm{C}_{23} \mathrm{H}_{25} \mathrm{~N}_{2} \mathrm{O}([\mathrm{M}+\mathrm{H}]+)$ : 345.1961 , found: 345.1964 .

$\mathrm{N}$-Benzyl-2-(benzylamino)-2-(4-chlorophenyl)acetamide (5m). ${ }^{1} \mathrm{H}$ NMR $\left(\mathrm{CDCl}_{3}\right) \delta 1.80$ (brs, $1 \mathrm{H}), 3.74$ (dd, 2H, $J=13,16 \mathrm{~Hz}), 4.26(\mathrm{~s}, 1 \mathrm{H}), 4.44$ (d, 2H, $J=6.2 \mathrm{~Hz}), 7.20-7.33$ (m, 14H), 7.42 (brs, $1 \mathrm{H}) ;{ }^{13} \mathrm{C} \mathrm{NMR}\left(\mathrm{CDCl}_{3}\right) \delta 43.3,52.4,66.2,127.5,127.6,127.7,128.2,128.7,128.7$, 128.7, 129.0, 134.0, 137.6, 138.2, 138.9, 171.5; IR (neat) 3304, 1658, 1517, 1089, 697; HRMS (FAB) calcd for $\mathrm{C}_{22} \mathrm{H}_{22} \mathrm{ClN}_{2} \mathrm{O}([\mathrm{M}+\mathrm{H}]+)$ : 365.1415 , found: 365.1420 .

$\boldsymbol{N}$-Benzyl-2-(benzylamino)-2-(furan-2-yl)acetamide (5n). ${ }^{1} \mathrm{H} \mathrm{NMR}\left(\mathrm{CDCl}_{3}\right) \delta 2.01$ (brs, $\left.1 \mathrm{H}\right)$, $3.47(\mathrm{~d}, 1 \mathrm{H}, J=13 \mathrm{~Hz}), 3.80$ (d, 1H, $J=13 \mathrm{~Hz}$ ), 4.39 (s, 1H), 4.49 (d, 2H, $J=6.2 \mathrm{~Hz}$ ), 6.30-6.36 $(\mathrm{m}, 2 \mathrm{H}), 7.23-7.38(\mathrm{~m}, 11 \mathrm{H}), 7.56$ (brs, $1 \mathrm{H}) ;{ }^{13} \mathrm{C} \mathrm{NMR}\left(\mathrm{CDCl}_{3}\right) \delta 43.3,52.2,60.2,108.4,110.5$, 127.4, 127.4, 127.6, 128.2, 128.6, 138.1, 138.8, 142.4, 151.4, 169.7; IR (neat) 3307, 1663, 1517 , 738; HRMS (FAB) calcd for $\mathrm{C}_{20} \mathrm{H}_{21} \mathrm{~N}_{2} \mathrm{O}_{2}([\mathrm{M}+\mathrm{H}]+)$ : 321.1598 , found: 321.1618 .

$\boldsymbol{N}$-Benzyl-2-phenyl-2-(phenylamino)acetamide (5o). ${ }^{5 \mathrm{c}}{ }^{1} \mathrm{H} \mathrm{NMR}\left(\mathrm{CDCl}_{3}\right) \delta 4.39(\mathrm{dd}, 1 \mathrm{H}, J=$ 5.5, $14 \mathrm{~Hz}), 4.54(\mathrm{~m}, 2 \mathrm{H}), 4.80(\mathrm{~s}, 1 \mathrm{H}), 6.64(\mathrm{~m}, 2 \mathrm{H}), 6.81(\mathrm{~m}, 1 \mathrm{H}), 7.00$ (brs, 1H), 7.13-7.45 (m, $12 \mathrm{H}) ;{ }^{13} \mathrm{C} \mathrm{NMR}\left(\mathrm{CDCl}_{3}\right) \delta 43.4,64.3,113.9,119.2,127.4,127.5,127.5,128.6,129.2,129.3$, $137.9,138.8,146.5,171.2$.

$N$-Benzyl-2-cyclohexyl-2-(phenylamino)acetamide (5p). ${ }^{1} \mathrm{H}$ NMR $\left(\mathrm{CDCl}_{3}\right) \delta$ 1.14-1.40 (m, $5 \mathrm{H}), 1.72-1.84(\mathrm{~m}, 5 \mathrm{H}), 2.15(\mathrm{~m}, 1 \mathrm{H}), 3.63(\mathrm{t}, 1 \mathrm{H}, J=4.1 \mathrm{~Hz}), 3.97(\mathrm{~s}, 1 \mathrm{H}), 4.38(\mathrm{dd}, 1 \mathrm{H}, J=$ $6.2,15 \mathrm{~Hz}), 4.49(\mathrm{dd}, 1 \mathrm{H}, J=6.2,15 \mathrm{~Hz}), 6.61(\mathrm{~m}, 2 \mathrm{H}), 6.79(\mathrm{~m}, 1 \mathrm{H}), 7.09-7.27(\mathrm{~m}, 7 \mathrm{H}) ;{ }^{13} \mathrm{C}$ NMR $\left(\mathrm{CDCl}_{3}\right) \delta 26.0,26.1,26.2,28.1,30.2,41.1,43.1,64.8,113.6,118.9,127.3,127.5,128.5$, 129.3, 138.1, 147.3, 172.6; IR (neat) 3302, 3056, 1646, 1602, 1507, 746; HRMS (FAB) calcd for $\mathrm{C}_{21} \mathrm{H}_{27} \mathrm{~N}_{2} \mathrm{O}([\mathrm{M}+\mathrm{H}]+)$ : 323.2118, found: 323.2119 .

2-((R)-1-Phenylethylamino)- $\boldsymbol{N}$-benzyl-2-phenylacetamide (5q). ${ }^{1} \mathrm{H} \mathrm{NMR}\left(\mathrm{CDCl}_{3}\right)$ [mixture] $\delta$ $1.35(\mathrm{~m}, 3 \mathrm{H}), 2.04$ (brs, 1H), $3.66(\mathrm{q}, 0.6 \mathrm{H}, J=6.9 \mathrm{~Hz}), 3.77$ (q, 0.4H, $J=6.9 \mathrm{~Hz}), 4.07$ (s, 
$0.4 \mathrm{H}), 4.11(\mathrm{~s}, 0.6 \mathrm{H}), 4.34(\mathrm{ddd}, 1.3 \mathrm{H}, J=6.2,15,32 \mathrm{~Hz}), 4.50$ (ddd, 0.7H, $J=6.2,15,32 \mathrm{~Hz}$ ), 7.14-7.36 (m, $15 \mathrm{H}), 7.59$ (brs, $1 \mathrm{H}) ;{ }^{13} \mathrm{C} \mathrm{NMR}\left(\mathrm{CDCl}_{3}\right)$ [mixture] $\delta 24.0,24.0,43.2,43.2,56.0$, $57.3,64.9,65.0,126.5,126.7,127.1,127.2,127.3,127.4,127.6,127.6,127.7,128.0,128.0$, 128.6, 128.6, 128.6, 128.7, 128.8, 128.8, 138.2, 138.4, 139.6, 139.7, 144.3, 144.3, 172.1, 172.4; IR (neat) 3300, 1658, 1518, 698; HRMS (FAB) calcd for $\mathrm{C}_{23} \mathrm{H}_{25} \mathrm{~N}_{2} \mathrm{O}\left([\mathrm{M}+\mathrm{H}]^{+}\right)$: 345.1961 , found: 345.1984 .

2-((R)-1-(Naphthalen-1-yl)ethylamino)- $N$-benzyl-2-phenylacetamide (5r). ${ }^{1} \mathrm{H}$ NMR $\left(\mathrm{CDCl}_{3}\right)$ [mixture] $\delta 1.50(\mathrm{~m}, 3 \mathrm{H}), 2.27(\mathrm{brs}, 1 \mathrm{H}), 4.15(\mathrm{~s}, 0.2 \mathrm{H}), 4.24(\mathrm{~s}, 0.8 \mathrm{H}), 4.30-4.35(\mathrm{~m}, 1.8 \mathrm{H}), 4.69$ $(\mathrm{m}, 0.2 \mathrm{H}), 7.06(\mathrm{~m}, 1 \mathrm{H}), 7.20-7.97(\mathrm{~m}, 15 \mathrm{H}) ;{ }^{13} \mathrm{C} \mathrm{NMR}\left(\mathrm{CDCl}_{3}\right)$ [mixture] $\delta 23.6,23.9,43.2$, $43.3,51.9,52.5,65.2,65.2,122.4,122.6,122.8,123.2,125.5,125.6,125.7,125.9,126.1,127.2$, $127.3,127.5,127.6,127.7,127.8,128.1,128.6,128.7,128.9,129.0,131.1,131.3,134.0,138.1$, 138.4, 139.7, 140.0, 140.2, 172.2, 172.6; IR (neat) 3309, 3057, 1653, 1510, 1454, 779, 699; HRMS (FAB) calcd for $\mathrm{C}_{27} \mathrm{H}_{27} \mathrm{~N}_{2} \mathrm{O}([\mathrm{M}+\mathrm{H}]+)$ : 395.2118 , found: 395.2137 .

2-((R)-1-(Naphthalen-2-yl)ethylamino)- $\boldsymbol{N}$-benzyl-2-phenylacetamide (5s). ${ }^{1} \mathrm{H} \mathrm{NMR}\left(\mathrm{CDCl}_{3}\right)$ [mixture] $\delta 1.43(\mathrm{~m}, 3 \mathrm{H}), 2.18(\mathrm{brs}, 1 \mathrm{H}), 3.83(\mathrm{q}, 0.6 \mathrm{H}, J=6.9 \mathrm{~Hz}), 3.94(\mathrm{q}, 0.4 \mathrm{H}, J=6.9 \mathrm{~Hz})$, $4.09(\mathrm{~s}, 0.4 \mathrm{H}), 4.15(\mathrm{~s}, 0.6 \mathrm{H}), 4.28(\mathrm{dd}, 0.3 \mathrm{H}, J=6.2,15 \mathrm{~Hz}), 4.32(\mathrm{dd}, 0.3 \mathrm{H}, J=6.2,15 \mathrm{~Hz})$, $4.48(\mathrm{dd}, 0.2 \mathrm{H}, J=6.2,14 \mathrm{~Hz}), 4.57(\mathrm{dd}, 0.2 \mathrm{H}, J=6.2,14 \mathrm{~Hz}), 7.10(\mathrm{~m}, 1 \mathrm{H}), 7.21-7.39(\mathrm{~m}, 9 \mathrm{H})$, 7.46-7,48 (m, 2H), $7.62(\mathrm{~m}, 1 \mathrm{H}), 7.79-7.82(\mathrm{~m}, 3 \mathrm{H}) ;{ }^{13} \mathrm{C} \mathrm{NMR}\left(\mathrm{CDCl}_{3}\right)$ [mixture] $\delta 24.0,24.0$, 43.2 , 43.3, 56.1, 57.4, 65.0, 65.0, 124.2, 124.6, 125.5, 125.8, 126.2, 127.2, 127.3, 127.5, 127.6, $127.7,128.0,128.1,128.5,128.6,128.7,128.8,128.9,132.9,133.3,133.4,18.2,138.4,139.6$, 139.6, 141.6, 141.7, 172.0, 172.4; IR (neat) 3314, 1660, 1600, 748, 698; HRMS (FAB) calcd for $\mathrm{C}_{27} \mathrm{H}_{27} \mathrm{~N}_{2} \mathrm{O}([\mathrm{M}+\mathrm{H}]+)$ : 395.2118 , found: 395.2131 .

$\boldsymbol{N}$-Phenyl-1-(phenylamino)cyclohexanecarboxamide (7). ${ }^{1} \mathrm{H} \mathrm{NMR}\left(\mathrm{CDCl}_{3}\right) \delta$ 1.25-1.39 (m, $3 \mathrm{H}), 1.63-1.68(\mathrm{~m}, 3 \mathrm{H}), 1.99-2.10(\mathrm{~m}, 4 \mathrm{H}), 4.10$ (brs, $1 \mathrm{H}), 6.68$ (d, 2H, $J=7.8 \mathrm{~Hz}), 6.83(\mathrm{~m}$, 1H), 7.08-7.29 (m, 5H), $7.51(\mathrm{~d}, 2 \mathrm{H}, J=7.8 \mathrm{~Hz}), 9.00(\mathrm{~s}, 1 \mathrm{H}) ;{ }^{13} \mathrm{C} \mathrm{NMR}\left(\mathrm{CDCl}_{3}\right) \delta 21.1,25.0$, 31.0, 61.1, 116.7, 119.7, 119.7, 124.0, 128.9, 129.2, 138.0, 143.7, 174.6; IR (KBr) 3418, 3349, 2947, 2851, 1668, 1607, 1515, 1433, 1303, 1183, 757, 697; HRMS (ESI-TOF) calcd for $\mathrm{C}_{19} \mathrm{H}_{23} \mathrm{~N}_{2} \mathrm{O}([\mathrm{M}+\mathrm{H}]+)$ : 295.1805, found: $295.1811 ; \mathrm{Mp}: 137-139{ }^{\circ} \mathrm{C}$.

Compound 9. ${ }^{1} \mathrm{H}$ NMR $\left(\mathrm{CDCl}_{3}\right) \delta 1.44(\mathrm{~m}, 1 \mathrm{H}), 1.69(\mathrm{~m}, 1 \mathrm{H}), 2.90-3.04(\mathrm{~m}, 3 \mathrm{H}), 3.12(\mathrm{td}, 1 \mathrm{H}$, $J=3.7,13 \mathrm{~Hz}), 3.16-3.26(\mathrm{~m}, 2 \mathrm{H}), 4.13(\mathrm{dd}, 2 \mathrm{H}, J=13,49 \mathrm{~Hz}), 5.12(\mathrm{~s}, 1 \mathrm{H}), 5.38(\mathrm{~m}, 1 \mathrm{H})$, 6.82-7.67 (m, 28H), $8.66(\mathrm{t}, 1 \mathrm{H}, J=13 \mathrm{~Hz}) ;{ }^{13} \mathrm{C} \mathrm{NMR}\left(\mathrm{CDCl}_{3}\right) \delta 28.8,32.6,34.1,36.0,57.8$, $72.3,78.2,89.1,122.1,123.1,123.4,125.4,126.1,127.2,127.3,127.4,127.7,128.1,128.2$, $128.4,128.5,128.7,129.1,129.2,129.7,129.8,130.5,131.0,134.3,134.4,138.5,139.5,141.1$, $141.4,142.9,161.7,164.7,166.0,167.2$; IR (KBr) 3509, 3393, 3061, 3030, 2948, 2920, 2861, 2830, 1789, 1704, 1632, 1602, 1452, 1373, 1341, 1298, 1231, 1218, 1073, 1055, 877, 746, 709; MS m/z $736([\mathrm{M}+\mathrm{H}]+)$; Anl. Calcd for $\mathrm{C}_{48} \mathrm{H}_{41} \mathrm{~N}_{5} \mathrm{O}_{3}$ : C, 78.34; H, 5.62; N, 9.52. Found: C, 78.16; H, 5.76; N, 9.45; Mp: $200-203{ }^{\circ} \mathrm{C}$. 
5-((Benzylamino)(phenyl)methyl)-5-hydroxy-1-methylpyrrolidin-2-one (11a). $\quad{ }^{1} \mathrm{H} \quad \mathrm{NMR}$ $\left(\mathrm{CDCl}_{3}\right)$ [major isomer] $\delta 0.99(\mathrm{dt}, 2 \mathrm{H}, J=10,16 \mathrm{~Hz}), 1.70(\mathrm{dt}, 1 \mathrm{H}, J=10,16 \mathrm{~Hz}), 1.94$ ( ddd, $1 \mathrm{H}, J=3,10,16 \mathrm{~Hz}), 2.39(\mathrm{~m}, 1 \mathrm{H}), 2.87(\mathrm{~s}, 3 \mathrm{H}), 3.56$ (d, 1H, $J=12 \mathrm{~Hz}), 3.77$ (d, 1H, $J=12$ $\mathrm{Hz}), 3.91(\mathrm{~s}, 1 \mathrm{H}), 7.26-7.36(\mathrm{~m}, 10 \mathrm{H})$, [minor isomer] $\delta 1.60(\mathrm{~m}, 1 \mathrm{H}), 1.75(\mathrm{~m}, 1 \mathrm{H}), 2.14(\mathrm{~m}$, $1 \mathrm{H}), 2.40(\mathrm{~m}, 1 \mathrm{H}), 2.79(\mathrm{~s}, 3 \mathrm{H}), 3.44(\mathrm{~d}, 1 \mathrm{H}, J=13 \mathrm{~Hz}), 3.72(\mathrm{~d}, 1 \mathrm{H}, J=13 \mathrm{~Hz}), 7.21-7.39$ (m, $10 \mathrm{H}) ;{ }^{13} \mathrm{C} \mathrm{NMR}\left(\mathrm{CDCl}_{3}\right)$ [major isomer] $\delta$ 25.0, 29.5, 29.9, 51.3, 65.0, 92.4, 127.2, 127.4, 128.2, $128.5,128.6,137.6,139.5,174.3$, [minor isomer] $\delta 25.5,29.1,30.4,51.4,68.1,92.0,127.3$, $127.3,128.3,128.5,128.6,128.7,137.1,129.3,175.2$; IR (KBr) [major isomer] 3331, 3080, 1666, 1603, 1449, 1184, 1102, 1023, 966, 728, 694; [minor isomer] 3304, 3165, 1647, 1666, 1541, 1398, 1113, 1077, 1023, 722, 697; HRMS (FAB) calcd for $\mathrm{C}_{19} \mathrm{H}_{23} \mathrm{~N}_{2} \mathrm{O}_{2}([\mathrm{M}+\mathrm{H}]+)$ : 311.1760, found: 311.1769; Mp: [major isomer] $122-125^{\circ} \mathrm{C}$, [minor isomer] $119-123{ }^{\circ} \mathrm{C}$.

3-((Benzylamino)(phenyl)methyl)-3-hydroxy-2-methylisoindolin-1-one (11b). ${ }^{1} \mathrm{H} \quad \mathrm{NMR}$ $\left(\mathrm{CDCl}_{3}\right)$ [diastereo mixture] $\delta 2.79(\mathrm{~s}, 0.44 \mathrm{H}), 2.84(\mathrm{~s}, 2.56 \mathrm{H}), 3.43-4.72(\mathrm{~m}, 2 \mathrm{H}), 4.20(\mathrm{~s}$, $0.15 \mathrm{H}), 4.23(\mathrm{~s}, 0.85 \mathrm{H}), 4.99(\mathrm{brs}, 1 \mathrm{H}), 6.75-7.88(\mathrm{~m}, 14 \mathrm{H}) ;{ }^{13} \mathrm{C}$ NMR $\left(\mathrm{CDCl}_{3}\right)$ [diastereo mixture] $\delta 24.3,25.5,51.6,66.5,67.5,77.2,91.1,122.5,122.7,122.8,124.0,127.2,127.8$, 127.9, 128.2, 128.3, 128.4, 129.4, 131.2, 131.3, 131.6, 131.6, 136.1, 139.4, 145.2, 167.6, 167.7; IR (KBr) 3250, 1674, 1438, 1394, 733, 718, 697; FABMS m/z $359([\mathrm{M}+\mathrm{H}]+)$; Anal. Calcd for $\mathrm{C}_{23} \mathrm{H}_{22} \mathrm{~N}_{2} \mathrm{O}_{2}$ : N, 7.82; C, 77.07; H, 6.19. found: N, 7.70; C, 76.82; H, 6.33.

\section{Acknowledgements}

This work was partially supported by a Grant-in-Aid for Scientific Research from Japan Society of the Promotion of Science (JSPS). 


\section{References and Notes}

1. Several examples of early transition metal imine complexes formed in situ: (a) Fukuhara, K.; Okamoto, S.; Sato, F. Org. Lett. 2003, 5, 2145. (b) Gao, Y.; Yoshida, Y.; Sato, F. Synlett 1997, 1353. (c) Honda, T.; Satoh, S.-I.; Mori, M. Organometallics 1995, 14 , 1548. (d) Coles, N.; Harris, M. C. J.; Whitby, R. J.; Blagg, J. Organometallics 1994, 33, 190. (e) Ito, H.; Taguchi, T.; Hanzawa, Y. Tetrahedron Lett. 1992, 33, 4469. (f) Grossman, R. B.; Davis, W. M.; Buchwald, S. L. J. Am. Chem. Soc. 1991, 113, 2321. (g) Jensen, M.; Livinghouse, T. J. Am. Chem. Soc. 1989, 111, 4495. (h) Wolczanski, P. T.; Bercaw, J. E. J. Am. Chem. Soc. 1979, 101, 6450.

2. Two-electron reduction of imine molecules with $\mathrm{SmI}_{2}$ or alkali metals under aprotic conditions: (a) Imamoto, T.; Nishimura, S. Chem. Lett. 1990, 1141. (b) Mehrotra, K. N.; Giri, B. P. Synthesis 1977, 489. (c) Mikhailov, B. M.; Kurdyumova, K. N. Zh. Obshch. Khim. 1958, 28, 355; Chem. Abstr. 1958, 52, 13685.

3. Reactions of Nb-imine, Ta-imine complexes: (a) Galakhov, M. V.; Gómez, M.; GómezSal, P.; Velasco, P. Organometallics 2005, 24, 848. (b) Bonanno, J. B.; Veige, A. S.; Wolczanski, P. T.; Lobkovsky, E. B. Inorganica Chimica Acta 2003, 345, 173. (c) Gómez, M.; Gómez-Sal, P.; Jiménez, G.; Martín, A.; Royo, P.; Sánchez-Nieves J. Organometallics 1996, 15, 3579. (d) Roskamp, E. J.; Dragovich, P. S.; Hartung, J. B., Jr.; Pedersen, S. F. J. Org. Chem. 1989, 54, 4736. (e) Roskamp, E. J.; Pedersen, S. F. J. Am. Chem. Soc. 1987, 109, 6551. (f) Chamberlain, L. R.; Rothwell, I. P.; Huffman, J. C. J. Chem. Soc., Chem. Commun. 1986, 1203. (g) Mayer, J. M.; Curtis, C. J.; Bercaw, J. E. J. Am. Chem. Soc. 1983, 105, 2651. (h) Takahashi, Y.; Onoyama, N.; Ishikawa, Y.; Motojima, S.; Sugiyama, K. Chem. Lett. 1978, 525.

4. For isolation of metal-imine complexes: (a) Zr: Scott, M. J.; Lippard, S. J. Organometallics 1997, 16, 5857. (b) Ti: Durfee, L. D.; Hill, J. E.; Fanwick, P. E.; Rothwell, I. P. Organometallics 1990, 9, 75. (c) Durfee, L. D.; Fanwick, P. E.; Rothwell, I. P.; Folting, K.; Huffman J. C. J. Am. Chem. Soc. 1987, 109, 4720. See also, 5c, 5d. (d) Nb: Richter, B.; Scholz, J.; Sieler, J.; Thiele, K.-H. Angew. Chem., Int. Ed. Engl. 1995, 34, 2649. (e) W: Legzdins, P.; Lundmark, P. J.; Rettig, S. J. Organometallics 1996, 15, 2988. (f) Chiu, K. W.; Jones, R. A.; Wilkinson, G.; Galas, A. M. R.; Hursthouse, M. B. J. Am. Chem. Soc. 1980, 102, 7978; J. Chem. Soc., Dalton. Trans. 1981, 2088.

5. (a) Cai, H.; Chen, T.; Wang, X.; Schultz, A. J.; Koetzle, T. F.; Xue, Z. Chem. Commun. 2002, 230. (b) Berno, P.; Gambarotta, S. Organometallics 1995, 14, 2159. (c) Gately, D. A.; Norton, J. R.; Goodson, P. A. J. Am. Chem. Soc. 1995, 117, 986. (d) Buchwald, S. L.; Watson, B. T.; Wannamaker, M. W.; Dewan, J. C. J. Am. Chem. Soc. 1989, 111, 4486.

6. (a) Clark, J. R.; Fanwick, P. E.; Rothwell, I. P. Organometallics 1996, 15, 3232. (b) Durfee, L. D.; Hill, J. E.; Fanwick, P. E.; Rothwell, I. P. Organometallics 1990, 9, 75. (c) 
Durfee, L. D.; Fanwick, P. E.; Rothwell, I. P. J. Am. Chem. Soc. 1987, 109, 4720. (d) Chiu, K. W.; Jones, R. A.; Wilkinson, G.; Galas, A. M. R.; Hursthouse, M. B. J. Chem. Soc., Dalton Trans. 1981, 2088.

7. Alexander, J. B.; Glueck, D. S. Organometallics 1995, 14, 3603.

8. (a) Takai, K.; Ishiyama, T.; Yasue, H.; Nobunaka, T.; Itoh, M. Organometallics 1998, 17 , 5128. (b) Makioka, Y.; Taniguchi, Y.; Fujiwara, Y.; Takaki, K.; Hou, Z.; Wakatsuki, Y. Organometallics 1996, 15, 5476.

9. For X-ray structure determination of niobium- or tantalum-imine complexes, see: (a) Figueroa, J. S.; Cummins, C. C. J Am. Chem. Soc. 2003, 125, 4020. (b) Mindiola, D. J.; Cummins, C. C. Organometallics 2001, 20, 3626. (c) Sánchez-Nieves, J.; Royo, P.; Pellinghelli, M. A.; Tiripicchio, A. Organometallics 2000, 19, 3161. (d) Boncella, J. M.; Cajigal, M. L.; Abboud, K. A. Organometallics 1996, 15, 1905. (e) Smith, D. P.; Strickler, J. R.; Gray, S. D.; Bruck, M. A.; Holmes, R. S.; Wigley, D. E. Organometallics 1992, 11, 1275. (f) Strickler, J. R.; Bruck, M. A.; Wigley, D. E. J. Am. Chem. Soc. 1990, 112, 2814. (g) Neithamer, D. R.; Párkányi, L.; Mitchell, J. F.; Wolczanski, P. T. J. Am. Chem. Soc. 1988, 110, 4421.

10. (a) Williams, R. M. In Synthesis of Optically Active $\alpha$-Amino Acids; Baldwin, J. E., Ed.; Organic Chemistry Series; Pergamon Press: Oxford, 1989. (b) Jacobsen, E. N.; Pfaltz, A.; Yamamoto, H. Comprehensive Asymmetric Catalysis; Springer: Berlin, 1999; Vols.1-3. (c) Ojima, I., Ed. Catalytic Asymmetric Synthesis, $2^{\text {nd }}$ Edn; Wiley-VCH: New York, 2000. (d) O'Donnell, M. J. Acc. Chem. Res. 2004, 37, 506, and references therein. (e) Maruoka, K.; Ooi, T. Chem. Rev. 2003, 103, 3013. (d) Arend, M. Angew. Chem., Int. Ed. 1999, 38, 2873. (e) Seebach, D.; Sting, A. R.; Hoffmann, M. Angew. Chem. Int. Ed. 1996, 35, 2708. (f) Duthaler, R. O. Tetrahedron 1994, 50, 1539. (g) Williams, R. M.; Hendrix, J. A. Chem. Rev. 1992, 92, 889.

11. A part of this work; Shimizu, H.; Ohsumi, M.; Kobayashi, S., Tetrahedron Lett. in press.

12. Recent reports on the synthesis of $\alpha$-amino- $\alpha, \alpha$-disubstituted acids, see: (a) Miyabe, H.; Asada, R.; Takemoto, Y. Tetrahedron 2005, 61, 385. (b) Belokon, Y. N.; Bespalova, N. B.; Churkina, T. D.; Císarová, I.; Ezernitskaya, M. G.; Harutyunyan, S. R.; Hrdina, R.; Kagan, H. B.; Kocovsky, P.; Kochetkov, K. A.; Larionov, O. V.; Lyssenko, K. A.; North, M.; Polásek, M.; Peregudov, A. S.; Prisyazhnyuk, V. V.; Vyskocil, S. J. Am. Chem. Soc. 2003, 125, 12860. (c) Ellis, T. K.; Martin, C. H.; Tsai, G. M.; Ueki H.; Soloschonok, V. A. J. Org. Chem. 2003, 68, 6208. (d) Clark, J. S.; Middleton, M. D. Org. Lett. 2002, 4, 765. (e) Fu, Y.; Hammarström, G. J.; Miller, T. J.; Froncezek, F. R. J. Org. Chem. 2001, 66,7118 .

13. Arai, S.; Sudo, Y.; Nishida, A. Synlett 2004, 1104.

14. Sugiura, M.; Kobayashi, S. Angew. Chem. Int. Ed. in press. 
15. Crystallographic data (excluding structure factors) for the structure reported in this paper have been deposited with the Cambridge Crystallographic Data Centre as supplementary publication no. CCDC-277587. Copies of the data can be obtained free of charge on application to CCDC, 12 Union Road, Cambridge CB2 1EZ, UK [fax.: (internat.) + 44 1223/336-033; e-mail: deposit@ccdc.cam.ac.uk].

16. Matsumoto, S.; Ishii, M.; Kimura, K.; Ogura, K. Bull. Chem. Soc. Jpn. 2004, 77, 1897. 